\title{
Detection and Identification of Pancreatic Cancer Using Probabilistic Neural Network
}

\author{
Ramya $\mathrm{N}^{\mathrm{a}, 1}$, Manoj Kumar $\mathrm{B}^{\mathrm{b}}$, Vijay Balaji $\mathrm{B}^{\mathrm{b}}$, Kiruthiga Devi $\mathrm{M}^{\mathrm{b}}$ and Deepika $\mathrm{R}^{\mathrm{c}}$ \\ al,c Assistant Professor, Dept of CSE, Sri Sairam Engineering College, India \\ ${ }^{b}$ UG Scholar, Dept of CSE, Sri Sairam Engineering College, Chennai, India
}

\begin{abstract}
The fact that pancreatic cancer has a low life expectancy, that is only $9 \%$ of people survive five years, makes a diagnosis catastrophic. The majority of patients are diagnosed late in life, where care choices are minimal. Early diagnosis of pancreatic cancer will greatly increase a person's chances of survival. Accurate PC staging will help doctors have the right treatment plan for PC patients at different stages, as well as the diagnostic measures needed for a quicker cancer recovery. In this proposed project, ultrasound images will be analyzed. The noise in the image is minimised using the Median Filter. In the next step, Gray Level Co-occurrence Matrix (GLCM) and Discrete Wavelet Transform (DWT)are used to extract related features .Following this extraction step, the refined characteristics are fed into a Probabilistic Neural Network (PNN) neural network classifier, which determines whether or not cancer is present. Metrics such as sensitivity, precision, and specificity are used in experimental computation.
\end{abstract}

Keywords. Pancreatic cancer, Ultrasound image, DWT, GLCM, PNN, Deep Learning.

\section{Introduction}

THE Pancreatic cancer is the tenth most common cancer in men and the ninth most common cancer in women in the US, however, it is the fourth most common cancer in both men and women. By 2020, pancreatic cancer is expected to lead lung cancer as the second leading cause of death in the US, demonstrating the disease's seriousness. However, India's annual pancreatic cancer burden was about 17,000 patients in 2008, and this number is projected to grow in the coming years. Multi centric research, a more comprehensive approach to reporting at all stages, and the identification of there have been potentially preventable risk factors for pancreatic cancer in the country India are all important in this situation. One of the main reasons for this is that there are no reliable early detection approaches for pancreatic tumors. Furthermore, the majority of the symptoms of a pancreatic tumor are unclear and may be caused by a number of other abdominal problems. Pain, weight reduction, conjunctivitis, loss of appetite, nausea, digestive changes, and diabetes are all some of the symptoms. After months of experiencing these symptoms ,most patients seek professional advice.

${ }^{1}$ Ramya N, Assistant Professor, Dept of CSE, Sri Sairam Engineering College, Chennai, India. E-mail: ramya.cse@sairam.edu.in 
As a result, most patients are diagnosed with a pancreatic tumors after it has progressed to the point that surgery is no longer an option. This is due to the fact that most large blood vessels are unable to be resected. Treatment becomes much more complex if the cancer has spread to other organs. As a result, there is an immediate need for a tool that will aid radiologists in the early detection of pancreatic tumors. The ultrasound images are used to process in this article. To remove the noise in the image, we use the Median Filter. DWT and GLCM are used to extract relevant features in the next step. The refined features are then fed into Probabilistic Neural Network (PNN) classifier which detects the cancer is present or not.

\section{Related Works}

[1] This paper was the first to implement an EL-SVM-based classification method involving pancreatic CT images. In terms of classification, the EL-SVM system performed best. The study will help to resolve some of the issues encountered in preoperative PC diagnosis and provide treatment options for different phases of PC patients which are achievable. It could not detect the early stage of the cancer.[2] In this paper, Because of the lethal nature of PDAC, it's important to rule out pancreatic cysts, which can be found in up to $16 \%$ of healthy people and can turn into PDAC. Pancreatic cysts come in a wide variety of sizes and forms, making accurate segmentation difficult, which limits for clinical manifestations, computer-aided analysis of CT images is being used. Using densely linked CNN, the author proposed, without pre-segmenting the condition, a framework for earlier staging and diagnosis of pancreatic tumour has been developed. The Dense-Net determine the significant features from the entire pathological pancreas and produces mappings between the appearance of pancreatic cysts on diagnostic devices and various pathological types of pancreatic cysts. They added feature vectors to the framework that helps physicians understand the deep learning method's decision, which improved facilitates business. On a population of 206 individuals with four psychotically identified subtypes of pancreatic tumour, the test had a prediction performance of $72.8 \%$, which is considerably high than the standard accuracy of $48.1 \%$. The high efficiency of the evolved approach on this complicated dataset obviously supports its therapeutic implications. [3] In this paper, HMI is an elasticity imaging technique used to measure tissue stiffness by measuring tissue dynamic displacements triggered by intermittent ultra - sonic radiation power. The aim of this study was to see if HMI could be used to detect pancreatic tumours and track HIFU high-intensity centred ultrasound care. The HMI system included a centred ultrasound transducer (FUS), which used diagnostic ultrasound transducer that used periodic radiation force to induce dynamic tissue movement at $50 \mathrm{~Hz}$ and $1 \mathrm{D}$ cross-correlation of acquired radiofrequency signals of ultrasound echoes to detect axial tissue displacement within the targeted region. HMI displacement images of cancer cells in a mouse model and healthy pancreases in a bizarre mouse model were constructed for pancreatic cancer classification. FUS was used to simultaneously induce HIFU thermal endoscopy and tissue motion for pancreatic tumour brachy therapy monitoring, allowing HMI monitoring without disrupting tumour endoscopy. Both pancreases were excised immediately after dilution for histological examination. Normal and malignant tissue were completely separated using HMI displacement images. The HMI monitoring shows significant pancreatic stiffening after 2 minutes of HIFU ablation, and histological analysis confirmed the development of thermal tumours. HMI was the first to prove that this could be used to 
detect pancreatic tumours and influence HIFU ablation in this research. It was also the first time an imaging technique centered on radiation force was used to control abdominal cancer HIFU diagnosis [7-11].

\section{Existing System}

- The existing system methodology is detecting the cancer using the CT (Computed Tomography) images.

- The LASSO algorithm (Least Absolute Shrinkage and Selection Operator) was chosen for feature selection of the images in it.

- It uses Ensemble Learning-Support Vector Machine (EL-SVM) as classifier to classify the tumour in pancreas.

- Accuracy of the system was about $85 \%$.

- It cannot give accurate results for the early stage of cancer.

\section{Proposed System}

- Ultrasound images are used for detecting the pancreatic cancer is present or not.

- Features are extractions of images are done using Gray Level Co-occurrence Matrix (GLCM) and Discrete Wavelet Transform (DWT).

- Probabilistic Neural Network (PNN) detects the cancer from the extracted features of input image and dataset images.

- It also identifies the stage of cancer whether it is benign or malign.

\section{Overview of The Project}

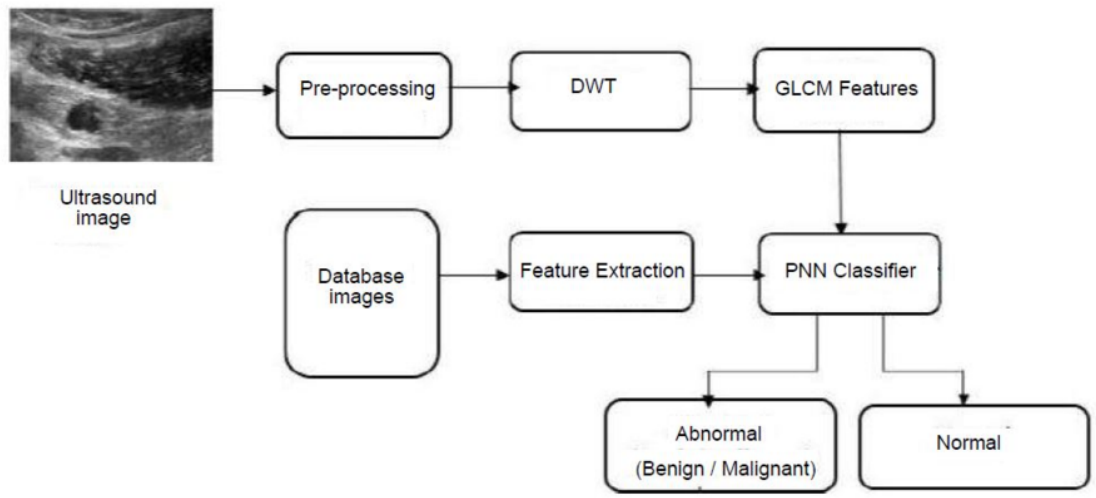

Figure 1. Architecture diagram of the project

At the first step, the given input image is preprocessed and then the feature of image are extracted using GLCM and DWT. Then the dataset is loaded and its features are extracted. The feature vector of the input and the dataset are given to the PNN classifier which detects and identifies the stage of cancer. 


\section{Implementation}

\subsection{Module 1: Preprocessing}

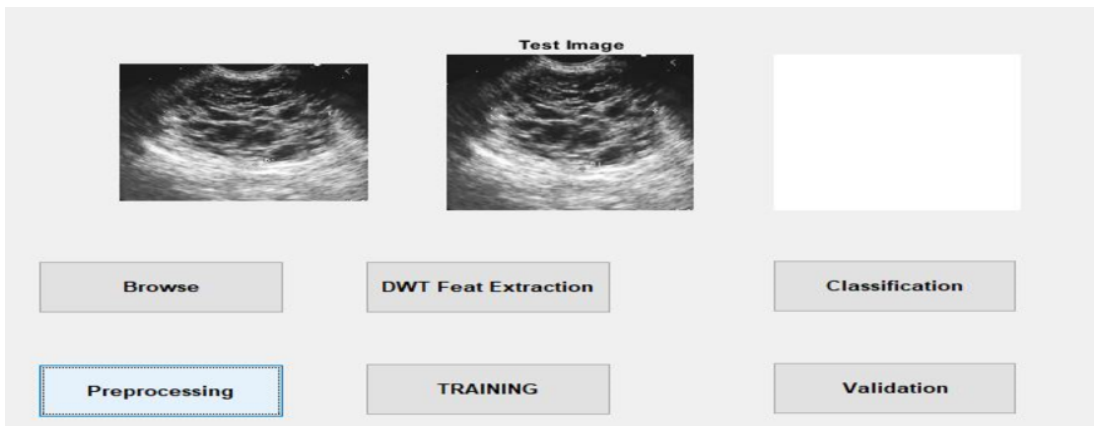

Figure 2.

Pre-processing is a technique for improving image data by eliminating unnecessary anomalies or optimizing image features that are needed for image acquisition. It accepts and generates intensity images. The method of estimating the clean original image from a corrupted/noisy image is called as image restoration.

\subsection{Module 2: Feature Extraction}

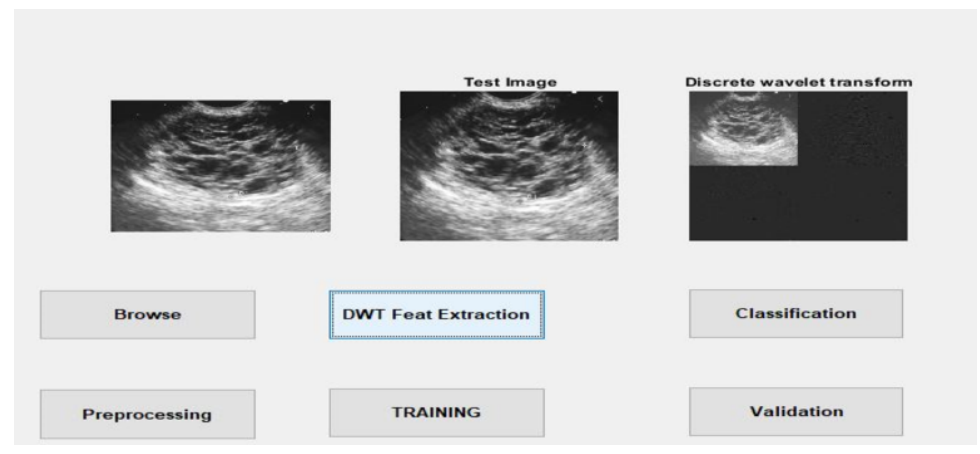

Figure 3.

Feature extraction is the method of extracting quantitative information from an image, such as colour features, texture, shape, and contrast. Wavelet coefficients were extracted using the discrete wavelet transform (DWT), and statistical features were extracted using the gray-level co-occurrence matrix (GLCM).

\subsection{Module 3 : Classification}

The PNN classifier classifies the input image by comparing with the dataset images and identifies whether cancer is present or not and also the stage of cancer. 


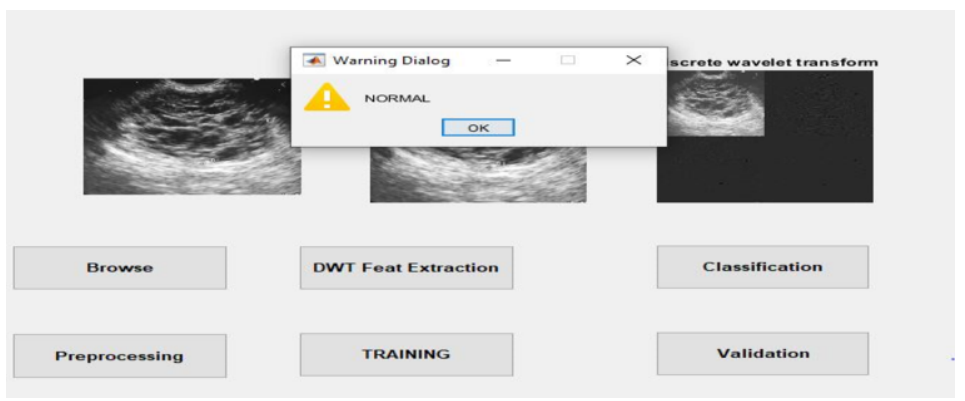

Figure 4.

\section{Conclusion}

It detects whether the cancer is present or not and if it is present then it identifies the stage of the cancer whether benign or malignant. The accuracy of this project is above $90 \%$. However, in future more samples can be given to the dataset and the accuracy can be increased.

\section{References}

[1] Min Li, Xiaohan Nie, Yilidan Reheman, Pan Huang, Shuailei Zhang, Yushuai Yuan, Chen Chen, Ziwei Yan. Computer-Aided Diagnosis and Staging of Pancreatic Cancer Based on CT images. IEEE, vol.8, July 2020.

[2] Bengio.Y, P. Simard, and P. Frasconi. Learning long-term dependencies with gradient descent is difficult. IEEE transactions on neural networks, 5(2):157-166, 1994.

[3] M.I.Canto, F. Harinck, R. H. Hruban, G. J. Offerhaus, J. W. Poley, I. Kamel, Y. Nio, R. S. Schulick, C. Bassi, I. Kluijt, M. J. Levy, A. Chak, P. Fockens, M. Goggins, and M. Bruno. International cancer of the pancreas screening (caps) consortium summit on themanagement of patients with increased risk for familial pancreatic cancer. Gut, 62(3):339-47, 2013.

[4] Daffa Fajri Riesaputri; Christy Atika Sari; Ignatius Moses Setiadi De Rosal; Eko Hari Rachmawanto. .Classification of Breast Cancer using PNN Classifier based on GLCM Feature Extraction and GMM Segmentation. IEEE, October 2020.

[5] Sachdeva J, Kumar V, Gupta I, Khandelwal N, Ahuja CK (2013) Segmentation, feature extraction, and multi class brain tumor classification. J Digit Imaging 26(6):1141-1150

[6] Demirhan A, Toru M, Guler I (2015) Segmentation of tumor and edema along with healthy tissues of brain using wavelets and neural networks. IEEE J Biomed Health Inform 19(4):1451-1458

[7] Hema Kumar.S, J.Uday Kiran, V.D.AKumar, G.Saranya, Ramalakshmi V .Effective Online Medical Appointment System. International Journal of Scientific \& Technology Research, Volume 8, Issue 09, September 2019, Pages $803-805$.

[8] Ambeth Kumar.V.D, Dr.M.Ramakrishnan, V.D.Ashok Kumar and Dr.S.Malathi (2015) .Performance Improvement using an Automation System for Recognition of Multiple Parametric Features based on Human Footprint .kuwait journal of science, Vol 42, No 1 (2015), pp:109-132.

[9] V.D.Ambeth Kumar, V.D.Ashok Kumar, S.Malathi, K.Vengatesan, M.Ramakrishnan .Facial Recognition System For Suspect Identification Using A Surveillance Camera . Pattern Recognition and Image Analysis (Springer), Volume 28, Issue 3, pp 410-420, 2018 . (DOI: 10.1134/S1054661818030136)

[10] Ambeth Kumar.V.D and M.Ramakrishan .Employment Of Footprint Recognition System . in the month of December for Indian Journal of Computer Science and Engineering (IJCSE) Vol. 3 No.6 Dec 2013

[11] Ramya,T.,Dr.Malathi,S.,ratheeksha,G.R. and Dr.V.D.Ambeth Kumar (2014).Personalized authentication procedure for restricted web service access in mobile phones.Applications of Digital Information and Web Technologies (ICADIWT), 2014, Page(s):69 - 74, Bangalore, India (ISBN:978-1-4799-2258-1) 